\title{
Embedded real-time system for detecting leakage of the gas used in iraqi kitchens
}

\author{
Husam Kareem \\ Directorate of Baghdad Education, Ministry of Education of Iraq, Iraq
}

\begin{abstract}
A major issue that happens in kitchens of houses and/or restaurants is the leakage of gas used as a fuel for cooker stove, which is commonly referred to as LPG (liquefied petroleum gas). LPG leakage may lead to a serious fire or even a deadly explosion that might affect the surrounding people. A substantial solution to avoid such disasters is by stopping its main cause. Therefore designing a device capable of monitoring and detecting such gases can minimize the dangerous and unwanted incidents by LPG leakage. This paper introduces a low cost and energy efficient real-time monitoring system that able to sense different dangerous gases, specifically those used for stove cooker. This system considers the pros of the previously introduced systems and fixes the cons available in those systems. In addition, the manufacturing cost has been taken into consideration. If the system senses any type of LPG gas (there is a gas leakage), it will react by making three different actions. It will make an alert sound to notify the people around the leakage place, send an SMS to two cell phones, and show, on an LCD screen, the leakage locationt.
\end{abstract}

Copyright (C) 2019 Institute of Advanced Engineering and Science. All rights reserved.

\section{Corresponding Author:}

Husam Kareem,

Directorate of Baghdad Education (Karkh 3),

Ministry of Education of Iraq, Iraq.

Email: husam_kf@yahoo.com

\section{INTRODUCTION}

In today's world, where most systems are becoming ubiquitous, the people safety plays a major role in all residential areas. Therefore, all expected, unwanted and dangerous, accidents that might happen should be avoided. One of the most likely happen incidents are fires and explosions because of LPG leakage. However, these accidents can be prevented by the early detection of its main cause (LPG leakage). It is worthy to mention that the LPG is a combination of highly flammable chemical such as propane and butane [1]. Nowadays, LP gas is widely used in houses for hot water, and food cooking. In addition, LPG has increasingly used as a second alternative fuel for cars. Hence, the usage of the introduced system device is not only limited to residential areas but also can be applicable in petrol stations that deals with such type of gases. Therefore, there is a growing necessity for a system that can detect the leakage and notify authorized people to fix it or at least keep it under control.

Besides the advantage of affordable cost, the system is designed to be highly scalable when there is a need to sense different and near locations within the communication range of RF technology (communication medium used in the design). In other words, the system can work as a single standalone device in one location or it can be a complete network in different locations with the same building (e.g. different apartments within the same residential building).

A real-time system is an attractive topic for both academic and industrial sections. Therefore, a wide variety of systems have been researched and created for different aspects of life like LPG monitoring systems [1], real-time sensing systems [2], healthcare system [3], smart lighting systems [4], tracking 
systems [5], and so on. Since this paper is introducing a real-time LPG monitoring and locating system, the work that has been introduced in the related scope will be explored in this section.

Majority of the existing LP Gas monitoring systems involves an alarm system, which is supposed to function upon gas sensing in houses and/or commercial locations. An embedded system for detecting hazardous gases is introduced in [6]. This system senses the surrounding area and if a hazardous gas is detected, it makes an alert and sends an SMS to the authorized person. Moreover, the system is designed using PIC microcontroller, which is not considered as contemporary device compared to the modern and open source microcontroller boards such as Arduino. A smart system for gas detection is designed to use in the area of that includes storage of natural gas is produced in [7]. The system measures the water and air quality using low-cost sensors and Arduino microcontroller. Although this system has reliable data, since it considers many factors during its measurements but because the system is designed for a specific environment with specific characteristics, it will not have the same performance in an environment that differs from the system's intended environment. Gas detection and monitoring system have introduced as embedded hardware prototype based on Android mobile platform [8]. This system using gas sensors to detect the leak of LP Gas and transmit the sensed data to the ATmega 16 microcontroller, and then the microcontroller will send the received data to an android mobile through Bluetooth module. The mobile is equipped with an application which is able to control the main switches of the location and able to control the exhaust fan. This system cannot consider as a reliable one since the leak might happen when the mobile is far from the microcontroller, which means that it is already out of range of communication. More precautions must be considered in such system since it is related to people safety and life. In addition, turning the ventilation fan most likely involves a spark that in return can make an explosion in the case of continuous leakage. This technical mistake is a serious drawback in this system. The same mistake has been included in a system that designed for LPG monitoring and automatic booking of the Gas cylinder [9]. The system included in its functionality that upon the leakage detection, besides notifying people, turning on the exhaust fan which, as mentioned above, could lead to a serious and immediate explosion.

In the design of any embedded system, different aspects should be taken into consideration, besides the system reliability and real-time response, the system cost is a significant factor. In other words, a perfect but expensive system could make it limited to rich institutes. On the other hand, a cheap, low-quality system is not the solution. Therefore, a reliable system and at the same time affordable for the majority of people who are in need of it is the best compromise. Because of the above-mentioned reasons, this system is meant to have an affordable value by the using low cost and open source microcontrollers that represent the system's core. Open source microcontroller is a kind of hardware that has unrestricted software and designs which is freely available for all users [10].

A number of LPG systems that have been introduced with the most optimized performance but they did not take in consideration minimizing the energy consumption as possible. Moreover, keeping the cost as low as possible which consequently lead to make it affordable for a higher number of end users. These two key indicators beside the performance indicators mentioned above are considered in the system introduced by this paper.

\section{THE PROPOSED METHOD}

In our daily lives, embedded systems can be easily fined everywhere. The number of embedded systems is rapidly increasing particularly in real-time monitoring systems. Moreover, the market of embedded systems is one of the fastest growing fields. As a definition, an embedded system is a dedicated purpose computing system designed to perform special functions [11].

Real-time systems are the systems that its computations reliability and robustness not only based on the accuracy of its result but also on its response time (the time when its results are issued) [8]. Real-time systems can be divided into two main categories: hard real-time and soft real-time systems. Hard real-time systems must always make a response to an event within the correct instant otherwise, it may lead to dangerous consequences on human lives, which means that the system has failed to be reliable. In contrast, soft real-time systems response time requirement is not as crucial as hard real-time. In other words, if the soft real-time system has failed to meet the deadline, it will not lead to a catastrophe [8].

As a result, the embedded systems that include real-time constraints are known as real-time embedded systems. In general, embedded and real-time systems consist of two main parts: software and hardware components. The hardware mainly relies on a microprocessor or a microcontroller attached to other devices such as I/O, LCDs, sensors, LEDs and so on. The software represents the driving force of the embedded real-time system. This software is usually has a specific application program and it is loaded to a ROM, EPROM, or Flash memory and when it is loaded, it will never change until it is required to be replaced or reloaded [11]. 
Monitoring system; some of them are related to performance aspects such as reliability, scalability, response time, portability and some others might have an economic aspect like fabrication cost. In addition, energy consumption can be considered the main factor that should be taken into consideration since it has a reflection on both sides: economic and performance. It can affect the performance directly because many real-time embedded systems are battery powered. On the other hand, it has an indirect effect on the economic aspect because in different developing countries, particularly in Iraq, energy conservation has become a great necessity since there is the inefficiency of electrical power [10].

The system introduced in this paper aim to decrease the fabrication cost by using open-source, low-cost devices. Furthermore, part of the main components of the system will operate only upon event detection (leakage of gas). Consequently, energy consumption by the entire system is reduced to the lowest possible level. In terms of reliability, the system utilized three means to notify the people in charge about the LP Gas leakage that are an LCD to show the leakage location, an alarm sound to notify the surrounding people, and a text message that send to two authorized people telling them that a gas leakage is happening and specify the leakage location. These three activities happen as a respond to LP Gas leak to guarantee a very high reliability for the system.

Based on the system design characteristics mentioned above, it can be clearly deduced that the system can achieve its design objectives, which are introducing a low-cost energy-efficient highly reliable and scalable embedded real-time system, used for LPG leakage detection.

\section{RESEARCH METHOD}

The prototype design and implementation processes of the proposed system is presented in this section. In general, any embedded system, in order to operate, requires an integration of two main parts, which are hardware and software. The hardware represents the physical architecture of the system, whereas the software involves the instructions and the protocols that drive and run the hardware components [10].

\subsection{Physical Architecture}

An open-source microcontroller platform is utilized to function as system's core. As we mentioned before, the system can operate in a single place as a single device or it can work as a complete sensor network that has different sensors distributed in different contiguous locations. Therefore, for the single detecting device, which works in only one location, an Arduino micro is used as the system core. On the other hand, when the system is designed as a complete sensor network, the system will have one central microcontroller represented by Arduino Mega and two or more microcontrollers represented by Arduino micro. In this case, the node that equipped with the Arduino Mega will function as the head (server) node for the entire network while the nodes that are equipped with Arduino micro will run as normal (ancillary) nodes. Moreover, a semiconductor type of sensors (MQ-6) is used in the system to play a major role in sensing and detecting the LP Gas concentrations in the air which mostly a composed of propane and butane [12]. Furthermore, being cheap makes this sensor more suitable for the development of low-cost embedded systems. The MQ-6 sensor is shown in Figure 1.

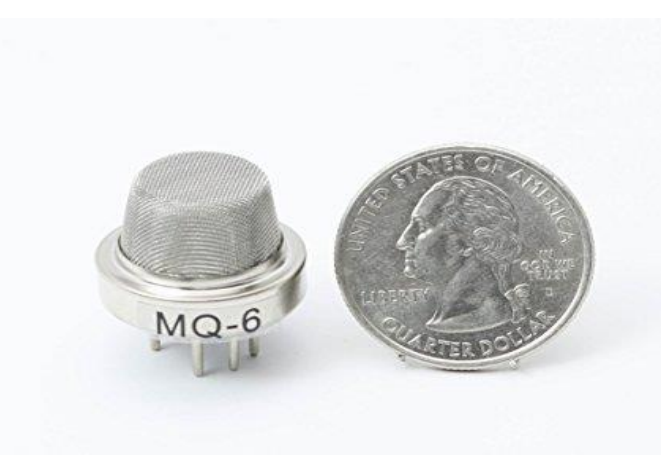

Figure 1. MQ-6 Gas sensor used for LPG detection

A GSM module, Piezo buzzer, and LCD are all equipped to the head node (the sensor node which is built on the Arduino Mega) to provide different options that notify the authorized persons upon LPG leakage detection. A block diagram that clarifies the flow of data through the normal sensor node is shown in Figure 2 and server sensor node is shown in Figure 3. 


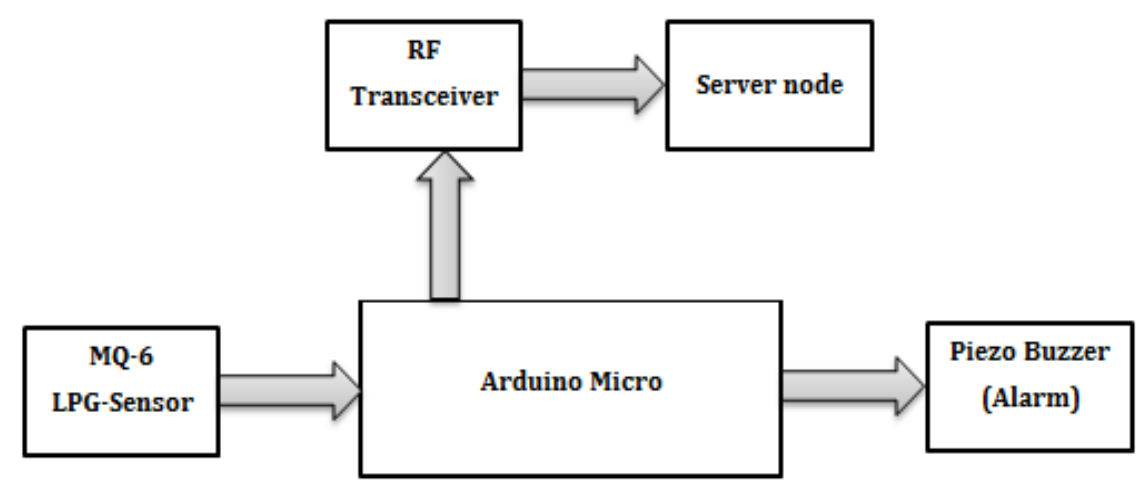

Figure 2. The data flow of the ancillary sensor node

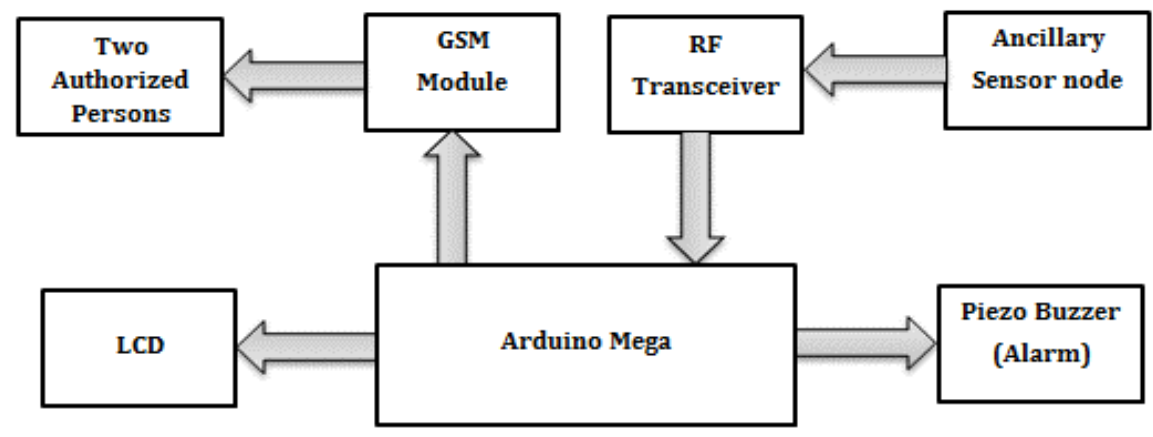

Figure 3. The data flow of the server sensor node

The system relies on Radio frequency (RF) technology as a communication medium to send/receive data between sensor nodes (ancillary nodes) and the head node (server node). RF used in many electronic applications since it does not require a line of sight.

\subsection{Software}

Since the main component of the system is the Arduino platform, which is controlling the operation of the entire system, therefore, the Arduino integrated development environment (IDE) is used to develop and evaluate the proposed system. In addition, different types of libraries that compatible with the Arduino IDE are utilized such as LCD and GSM. It is worthy to mention that this software is freely available which makes it very suitable for low-cost systems development.

\section{RESULTS AND DISCUSSION}

Foremost, the system is designed to be highly applicable for the residential buildings (number of units in the same vertical building). Therefore, as mentioned earlier, different contiguous locations can be equipped with sensor nodes and a central node need to be installed at the management office to monitor the entire building. A number of energy efficient routing algorithms for wireless sensor networks that have grid topology can be utilized to transmit the data from ancillary nodes to the server node such as algorithms in [13], [14] and [15].

In order to give a simple and clear image about the system operation, it will be divided into two operation modes: standby mode when there is no leak and the activation mode when a leakage is detected. During the standby mode, the MQ-6 sensor in each sensor node is monitoring the level of LPG concentration in the air. The RF module is sending nothing to the server node and the piezo is not activated.

Upon leakage detection, the piezo buzzer would be activated immediately as well as the RF module would communicate with the server node to notify the management that there is a leak in a specific location. 
Based on the mac address of each RF module that is equipped with the sensor nodes, the server node can recognize which unit that has a leakage. In return, the server node will show on its LCD which unit that has a leakage and at the same time, it will activate its piezo to notify the authorized people at the management office. Moreover, using the GSM module, it will send SMS messages to two pre-defined phone numbers (the numbers are preferred to be for the unit owners).

\subsection{Power Reduction Strategy}

In addition to using low energy consumption devices, a technique is used to minimize the power consumption as low as possible. The idea is that when there is no leak in LP GAS, there is no need to operate the piezo, even if it is in standby mode but it is still using some amount of current. Therefore, a transistor is used as a switch between each piezo and its corresponding microcontroller. Once a leak is detected, the transistor is activated and then the piezo is turned on making an alarm sound. By following this strategy, the energy consumption for the entire system is minimized to its lowest possible level. Figure 4 shows a schematic diagram of connecting the piezo to the transistor.

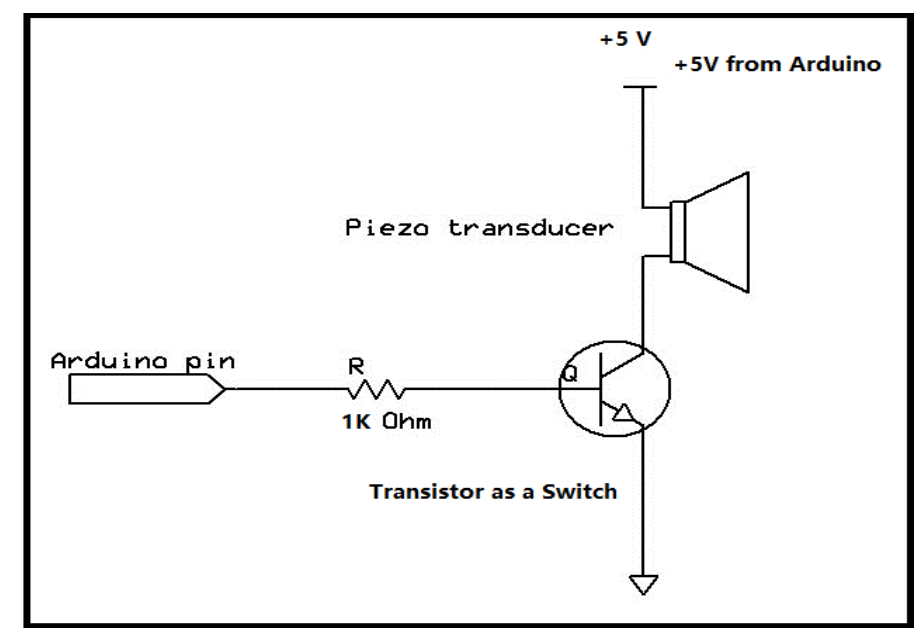

Figure 4. Transistor as a switch connected between the Arduino and the Piezo

\section{CONCLUSION}

The design and implementation process of a Low-cost LPG leakage real-time detection system is discussed in this research. Since the undetected leakage or even late detection is highly probable can lead to a serious explosion that might take lives. Therefore, such systems have earned a great necessity in vulnerable locations especially kitchens of houses and/or restaurants. Taking into consideration the importance of such system in all residential places. The presented system is developed using equipment with low cost and low energy consumption leading to make the system affordable for the majority of people. In addition, the system is highly scalable by adding more sensor nodes as ancillary nodes. Thus, this system outperforms other ordinary systems by overcoming the defects available in previous work, reducing the fabrication cost and energy consumption, being highly scalable using master /slave strategy between microcontrollers. Moreover, being highly scalable can add an extra advantage to the system besides monitoring more locations, the system can be developed easily to perform more functions since each sensor node is built based on its own microcontroller so adding more functions to the node leading to adding more functions to the entire system. As part of our future work, the system will be developed to include more functions such as anti-theft security sub-system, temperature and humidity monitoring and control.

\section{REFERENCES}

[1] Shrivastava, A., Prabhaker, R., Kumar, R., \& Verma, R. (2013). "GSM based gas leakage detection system", International Journal of Emerging Trends in Electrical and Electronics, Vol 1, No. 2, pp:42-45.

[2] Nabeel, S. A., Zaid, A. A. A., Abdulhussein, A. (2018). "Real-time Heart Pulse Monitoring Technique Using Wireless Sensor Network and Mobile Application". International Journal of Electrical and Computer Engineering (IJECE), Vol.8, No.6, pp:5118-5126. 
[3] Aziz, K., Tarapiah, S., Ismail, S. H., \& Atalla, S. (2016, March). Smart real-time healthcare monitoring and tracking system using GSM/GPS technologies. In Big Data and Smart City (ICBDSC), 2016 3rd MEC International Conference on (pp. 1-7). IEEE.

[4] Rajina, R. M., Mohamad, A. M., Arniyati, A., \& Muhamad, A. H. Abd Halim. (2018). "Provisioning of Street Lighting based on Ambience Intensity for Smart City", Indonesian Journal of Electrical Engineering and Computer Science, Vol. 12, No. 3, pp:1401-1406.

[5] Suresh, S.,Ahmad S. A. (2018). "Improvement of Auto-Tracking Mobile Robot based on HSI Color Model". Indonesian Journal of Electrical Engineering and Computer Science, Vol. 12, No.3., pp:1349-1357.

[6] Ramya, V., \& Palaniappan, B. (2012). Embedded system for Hazardous Gas detection and Alerting. International Journal of Distributed and Parallel Systems (IJDPS) Vol, 3, No. 3, PP:287-300.

[7] Ilie, A. M., \& Vaccaro, C. (2017, July). Design of a smart gas detection system in areas of natural gas storage. In Geoscience and Remote Sensing Symposium (IGARSS), 2017 IEEE International (pp. 5954-5957). IEEE.

[8] Kurzekar, R., Arora, H., \& Shrestha, R. (2017, December). Embedded Hardware Prototype for Gas Detection and Monitoring System in Android Mobile Platform. In Nanoelectronic and Information Systems (iNIS), 2017 IEEE International Symposium on (pp. 6-10). IEEE.

[9] Naik, R. N., Reddy, P. S. N., Kishore, S. N., \& Reddy, K. T. K. (2016). Arduino Based LPG gas Monitoring \& Automatic Cylinder booking with Alert System. IOSR Journal of Electronics and Communication Engineering (IOSR-JECE), Vol. 11, No. 4, pp: 6-12.

[10] Hadi Jameel, Husam Kareem," Low-Cost Energy-Efficient Smart Monitoring System Using Open-Source Microcontrollers”, International Review of Automatic Control (IREACO), Vol.9, No.6, (200X), pp.423-428.

[11] Qian K., den Haring D., Cao L. (2009) Introduction to Embedded Systems. In: Embedded Software Development with C. Springer, Boston, MA.

[12] The Spark fun website. [Online]. Available: http:// https://www.sparkfun.com/products/9405.

[13] Farhan, H. K. (2016). Enhanced Chain-Cluster Based Mixed Routing Algorithm for Wireless Sensor Networks. Journal of Engineering, Vol. 22, No. 1,PP:103-117.

[14] Kareem, H., \& Jameel, H. (2018, October). Maintain Load Balancing in Wireless Sensor Networks Using Virtual Grid Based Routing Protocol. In 2018 International Conference on Advanced Science and Engineering (ICOASE) (pp. 227-232). IEEE.

[15] Kareem, H., Hashim, S. J., Suberamaniam, S., \& Sali, A. (2014). Energy efficient two stage chain routing protocol (TSCP) for wireless sensor networks. J Theor Appl Inf Technol, Vol. 59, No.2, pp:442-450.

\section{BIOGRAPHIES OF AUTHORS}

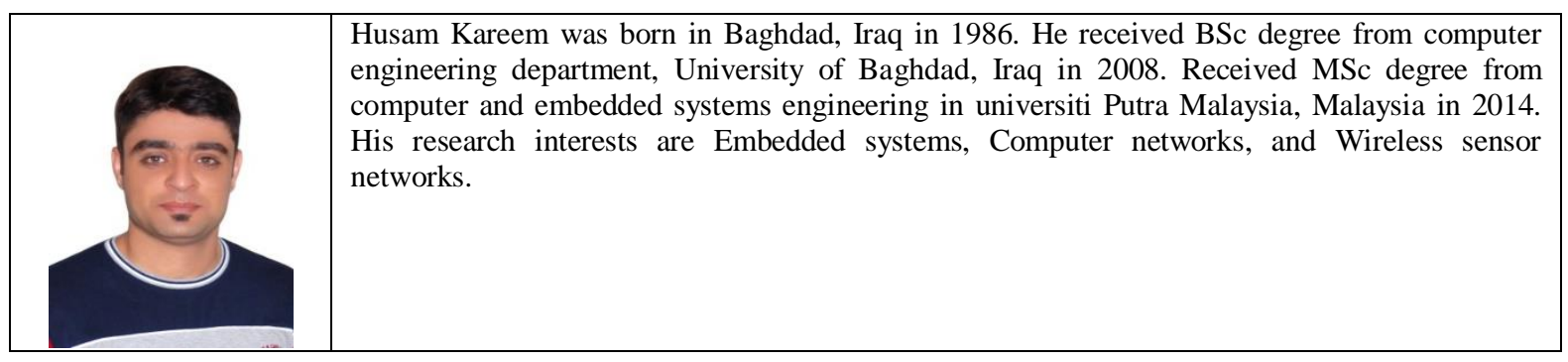

